\title{
Visualization of Hyperspectral Imaging Data Based on Manifold Alignment
}

\author{
Danping Liao \\ College of Computer Science \\ Zhejiang University, Hangzhou, China \\ liaodanping@gmail.com
}

\author{
Yuntao Qian \\ College of Computer Science \\ Zhejiang University, Hangzhou, China \\ ytqian@zju.edu.cn
}

\author{
Jun Zhou \\ School of ICT \\ Griffith University, Nathan, Australia \\ jun.zhou@griffith.edu.au
}

\begin{abstract}
Tristimulus display of the abundant information contained in a hyperspectral image is a challenging task. Previous visualization approaches focused on preserving as much information as possible in the reduced spectral space, but ended up with displaying hyperspectral images as false color images, which contradicts with human experience and expectation. This paper proposes a new framework to tackle this problem. It is based on the fusion of a hyperspectral image and a high-resolution color image via manifold alignment technique. Manifold learning is an important tool for dimension reduction. Manifold alignment projects a pair of two data sets into a common embedding space so that the pairs of corresponding points in these two data sets are pairwise aligned in this new space. Hyperspectral image and high-resolution color image have strong complementary properties due to the high spectral resolution in the former and the high spatial resolution in the latter. The embedding space produced by manifold alignment bridges a gap between the high dimensional spectral space of hyperspectral image and RGB space of color image, making it possible to transfer the natural color and spatial information of a high-resolution color image to a hyperspectral image to generate a visualized image with natural color distribution and finer details.
\end{abstract}

Keywords-Hyperspectral image, visualization, manifold alignment, image fusion

\section{INTRODUCTION}

A hyperspectral imaging sensor acquires images of the same area using hundreds of bands. It provides high-resolution spectral information such that more accurate material detection, classification, and quantification can be achieved. Hyperspectral image (HSI) displaying has been a challenging task because it contains much more bands than can be displayed on a tristimulus display device [1]. Although the requirements of HSI visualization are task dependent, there are some common goals such as information preservation, consistent rendering, edge salience, and natural palette [2]

HSI visualization can be seen as a specific dimension reduction problem where high dimensional spectral space of HSI is projected into RGB color space. A direct and simple type of visualization approach is to use interactive tools to manually pick three spectral bands from HSI and match them to RGB channels [3], or automatically select the important/informative bands based on various statistical measures [4]. More sophisticated mappings can be created through user-specified linear combinations of spectral bands [5], or data-dependent combinations using principle component analysis(PCA) [6]. All these methods are based on the assumption that the HSI data is embedded almost linearly in the ambient space. In recent years, some nonlinear dimension reduction methods are employed in HSI visualization and feature extraction, including manifold learning [7], Markov random field (MRF) fusion [8], nonlinear optimization [9], and bilateral filtering [10]. These approaches aim at discovering the nonlinear structure of the HSI, and demonstrating better performance in preserving the intrinsic information of HSI.

Unbounded three-dimensional Euclidean space produced by dimension reduction is normally not suitable for displaying because it does not considered the boundaries of the hue, saturation, and value (HSV) color space. In order to use the dynamic range of RGB color space, a post-processing transform is needed to map the original three-dimensional space to RGB space [9]. However, existing transforms render the image without taking into account the visual effect of color distribution. A common practice is using false color to display the dimension reduced HSI, which contradicts with human experience and expectation. Furthermore, while mapping the data to RGB space using techniques such as nonuniform scaling, standard deviation stretch, and histogram equalization, information in the original HSI may be distorted.

In this paper, we propose a HSI visualization method based on manifold alignment with the aid of a high resolution color image (HRCI). In most cases, we can acquire HSI and HRCI on the same spot by HSI/HRCI integrated or separated imaging sensors. The HSI and HRCI have strong complementary properties for information fusion. Although HSI contains precise and detailed spectral information, its spatial resolution is lower than HRCI images. On the other hand, HRCI has comparatively high spatial resolution. The fusion of different views of the same input data has been receiving increased attention [11]. The HSI and panchromatic image (PAN) fusion based visualization methods [12], [13] take advantage of the high spatial resolution of PAN in sharpening the HSI visualization. They are also called pan-sharpening algorithms, which are not limited to HSI applications [14], [15]. However, these pan-sharping methods require precise matching between HSI and PAN, which is not an easy task especially when HSI and PAN are acquired from different sensors such as airplanebased and satellite-based systems with different geometrical distortions. The main merit of the proposed method is that only a small number of corresponding pixel pairs between HRCI and HSI are needed rather than the precise registration between two images, which makes the algorithm very flexible.

Manifold alignment is one of the important frames for transfer learning [16]. It builds connections between two or 
more disparate data sets by aligning their underlying manifolds and enables knowledge transfer across the data sets [17], [18]. We treat HSI visualization with the help of HRCI as a transfer learning problem. The goal is to transfer the natural color distribution and fine spatial information of HRCI to HSI. To achieve this goal, these two types of images are connected by the corresponding pixels and are aligned to a common embedding space. The embedding space bridges the gap between the high dimensional space of HSI and RGB space of HRCI, making it possible to transfer the knowledge between them. Compared to other dimensionality reduction based HSI visualization methods, the proposed approach projects the HSI directly to RGB space, so that no additional technique is required to fit the dynamic range of the tristimulus display and human visual habit. Furthermore, mutual compensation of HSI and HRCI in spatial and spectral spaces is also embodied in the visualization result.

This paper is organized as follows. Section II provides the background knowledge of manifold alignment. In Section III, manifold alignment based HSI visualization approach are presented. The experiments and results are discussed in Section IV. Conclusions are drawn in Section V.

\section{Manifold Alignment}

Given two data sets that are represented by the matrices $S \in R^{n_{s} \times p_{s}}$ and $T \in R^{n_{t} \times p_{t}}$ where $n_{s}$ and $n_{t}$ are the numbers of samples, and $p_{s}$ and $p_{t}$ are the numbers of features. Assume that partial correspondence information are given in the form of paired samples $x_{s}^{i} \in S \leftrightarrow x_{t}^{j} \in T$. Manifold alignment uses the corresponding sample pairs between two data sets to find two transformation functions $f_{s}: R^{p_{s}} \rightarrow R^{q}$ and $f_{t}$ : $R^{p_{t}} \rightarrow R^{q}$, which leads to implicit alignment of different data manifolds in the mutual embedding space $R^{q}$.

To achieve this goal, we firstly construct the weighted graph that represents two input manifolds and their correspondences. In the graph of each data set, the nodes/samples $i$ and $j$ are connected by an edge if $i$ is among the $k$ nearest neighbors of $j$ or $j$ is among the $k$ nearest neighbors of $i$. The weight of an edge can be calculated by a heat kernel

$$
W(i, j)=e^{\frac{-\left\|x_{i}-x_{j}\right\|^{2}}{\sigma}}
$$

Now we combine these two individual graphs into a joint graph by constructing the edges between nodes $i$ and $j$ from different data sets. Typically, $W(i, j)=1$ if samples $x_{i}$ and $x_{j}$ form a corresponding pair. As a result, the size of the weighted adjacency matrix of this joint graph is $\left(n_{s}+n_{t}\right) \times\left(n_{s}+n_{t}\right)$, and the entries of $W$ are defined as

$$
W(i, j)= \begin{cases}\alpha_{1} W_{i j} & x_{i} \text { and } x_{j} \text { are from the same dataset } \\
\alpha_{2} W_{i j} & x_{i} \text { and } x_{j} \text { are corresponding pair } \\
& \begin{array}{l}
\text { from two datasets respectively } \\
0
\end{array}\end{cases}
$$

The cost function for manifold alignment is defined as

$$
\begin{array}{r}
H\left(f_{s}, f_{t}\right)=\sum_{i \neq j}\left\|f_{s}\left(x_{s}^{i}\right)-f_{s}\left(x_{s}^{j}\right)\right\|^{2} \times \alpha_{1} W_{s}(i, j) \\
+\sum_{m \neq n}\left\|f_{t}\left(x_{t}^{m}\right)-f_{t}\left(x_{t}^{n}\right)\right\|^{2} \times \alpha_{1} W_{t}(m, n) \\
+\sum_{i \neq m}\left\|f_{s}\left(x_{s}^{i}\right)-f_{t}\left(x_{t}^{m}\right)\right\|^{2} \times \alpha_{2} W_{s t}(i, m)
\end{array}
$$

In this equation, the first two terms on the right-hand side ensure that two similar samples from the same data set maintain their similarity in the common space, and the last term ensures that the corresponding pairs from different data sets stay close in the common space.

By constructing the joint adjacency graph $W$, two manifolds are treated as a joint manifold. The alignment goal turns to finding an optimal mapping function to reduce the dimensionality of the joint structure. Thus Equation (3) can be rewritten as a general loss function defined as

$$
H\left(f_{s t}\right)=\sum_{i \neq j}\left\|f_{s t}\left(x_{i}\right)-f_{s t}\left(x_{j}\right)\right\|^{2} W(i, j)
$$

where $f_{s t}$ is the joined function of $f_{s}$ and $f_{t}$ that are applied to data sets $S$ and $T$ respectively, and $W$ is the joint adjacency matrix. The summation is taken over all pairs of pixels from these two data sets. Equation (4) will derive a simplified form by the joint graph Laplacian $L=D-W$ where $D$ is a diagonal matrix with $D(i, i)=\sum_{j} W(i, j)$.

$$
H\left(f_{s t}\right)=\operatorname{tr}\left(F^{\prime} L F\right)
$$

where $F$ is an $\left(n_{s}+n_{t}\right) \times q$ matrix that represents two data sets in the embedding space, and $F^{\prime}$ is the transpose of $F$. When the manifold projection is linear, $f_{s}$ and $f_{t}$ are two transformation matrices $F_{s}$ and $F_{t}$ with the size of $p_{s} \times q$ and $p_{t} \times q$ respectively, i.e., $f_{s}\left(x_{i}\right)=x_{i} F_{s}$ and $f_{t}\left(x_{i}\right)=x_{i} F_{t}$. Therefore, the cost function for the linear manifold alignment is described as

$$
H\left(f_{s t}\right)=\sum_{i, j}\left\|x_{i} F-x_{j} F\right\|^{2} W(i, j)
$$

where $F=\left[F_{s} ; F_{t}\right]$ is the joint matrix of $F_{s}$ and $F_{t}$ with size $\left(p_{s}+p_{t}\right) \times q$. The cost function Equation (6) is simplified as

$$
H\left(f_{s t}\right)=\operatorname{tr}\left(F^{\prime} X^{\prime} L X F\right)
$$

where $X$ is is an $\left(n_{s}+n_{t}\right) \times\left(p_{s}+p_{t}\right)$ joint data matrix with $S$ on the upper left corner, $T$ on the lower right corner and zero on the other entries. This optimization problem can be solved by Laplacian eigenmaps, i.e., $F$ is constructed by the eigenvectors which correspond to the $q$ minimum eigenvalues of the generalized eigenvector problem.

$$
X^{\prime} L X \hat{F}=\lambda X^{\prime} D X \hat{F}
$$

Linear alignment sacrifices the ability to align arbitrarily warped manifolds, but it is much faster than nonlinear alignment. In this paper, linear method is used in all experiments. 


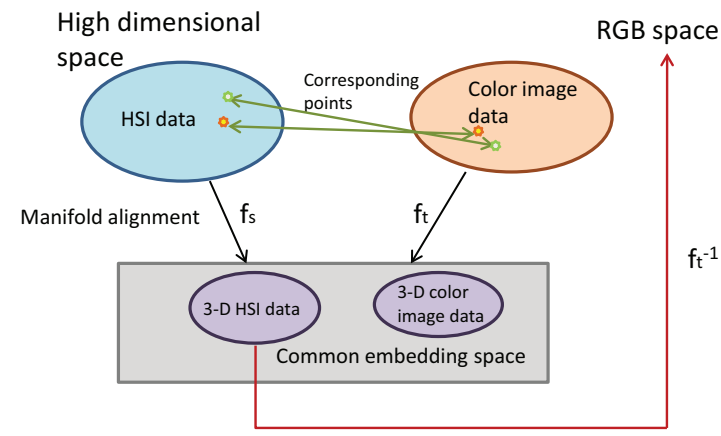

Fig. 1. The process of the proposed visualization method.

\section{Manifold Alignment Based Hyperspetral IMAGE VISUALIZATION}

Given an HSI with high spectral resolution but low spatial resolution and a HRCI with high spatial resolution in RGB color space, the main goal of our approach is to inject the HRCI's natural color as well as its spatial information into the visualized HSI to achieve better visual effects and finer detail information. Fig. 1 summarizes the process of the manifold alignment based visualization. Firstly, the corresponding pairs are found between a HSI and a HRCI that are acquired on the same spot, which builds the connection between the two manifolds from different spaces. Secondly, the manifold alignment aligns these two manifolds to a common embedding space by projection functions $f_{s}$ and $f_{t}$ respectively, where $f_{s}$ is the mapping function from HSI space to the embedding space and $f_{t}$ is the one from RGB space to the embedding space. Finally, the HSI in the embedding space is mapped to RGB space by the inverse projection function of $f_{t}$.

The overall visualization algorithm can be summarized as follows:

1) Create the weighted adjacency matrices $W_{s}$ and $W_{t}$ of HSI and HRCI using $k$ nearest neighbors and heat kernel.

2) Find the corresponding points between HSI and HRCI using SIFT based feature matching.

3) Construct the joint weighted adjacency matrix $W$ using $W_{s}$ and $W_{t}$ along with the corresponding pixelpairs, and then obtain its Laplacian matrix $L$.

4) Solve the optimization problem by eigenmaps to get the linear transformation matrices $F_{s}$ and $F_{t}$.

5) Calculate the mapping function $F_{t}^{-1}$ that is the inverse transform of $F_{t}$. It maps the data from the embedding space to RGB space.

6) Map the embedded HSI data to RGB space by $F_{t}^{-1}$.

Using the notations in section II, the HSI $I_{s}$ and HRCI $I_{t}$ are represented in their original spaces respectively by two matrices $S$ and $T$. The numbers of rows in $S$ and $T$ are $n_{s}$ and $n_{t}$, which are their image sizes respectively, and the numbers of columns of $S$ and $T$ are $p_{s}$ and $p_{t}$, which are their color dimensions respectively. Since HRCI is in RGB space, $p_{t}=3$.

In step 1, we use different distance measures to calculate the similarity between a pair of pixels for HSI and HRCI respectively. As the material reflectance or radiance differs in scale in different bands, the spectral angle distance (SAD) are commonly used to measure the difference of a pair of pixels in HSI. Thus, the weighted adjacency matrix $W_{s}$ of HSI is defined as

$$
W_{s}(i, j)=e^{\frac{-S A D\left(x_{i}, x_{j}\right)}{\sigma}}
$$

where

$$
S A D\left(x_{i}, x_{j}\right)=\arccos \left(\frac{x_{i} \cdot x_{j}}{\left\|x_{i}\right\|\left\|x_{j}\right\|}\right)
$$

As HRCI is in RGB space, $W_{t}$ is calculated by Equation (1) using Euclidean distance in the heat kernel.

Step 2 is to find some correspondences between the HSI and HRCI which are used to align two manifolds. The scaleinvariant feature transform (SIFT) feature is widely used to find the corresponding pixels due to its robustness under changes in scale, orientation and illumination [19], and has been applied for image registration and stitching. As SIFT keypoints are located at the extrema of difference of Gaussian (DoG), simply performing SIFT keypoint matching may lead to the result that the corresponding pixels are found only in the sharp regions. However, the color distribution in the homogeneous regions in HRCI such as water, flat ground, etc. are also essential to HSI visualization. On the other hand, only using SIFT feature to find the corresponding pixels is not precise, because the geometric structure of image is not taken into account. Here, we propose a two-stage method that first coarsely estimate an affine transform for image registration, and then finely match some pixels by neighborhood searching.

In the registration stage, an affine transformation matrix $\mathbf{H}$ with the size of $3 \times 3$ is estimated that coarsely maps each pixel in the HSI to a pixel in the HRCI [20].

$$
\left(x^{\prime}, y^{\prime}, a\right)^{T}=\mathbf{H}_{3 \times 3}(x, y, 1)^{T}
$$

where $(x, y)$ is a pixel coordinate in the HSI, and $\left(x^{\prime} / a, y^{\prime} / a\right)$ is its coarse corresponding pixel in the HRCI. To estimate $\mathbf{H}$, we firstly extract SIFT features for all spectral band images of HSI, and for all RGB channels of HRCI, respectively. Then the SIFT key points between HSI and HRCI are matched, and finally the transformation matrix $\mathbf{H}$ is estimated using least squares optimization and random sample consensus (RANSAC) based outlier removal. As we know, affine function is too simple to represent many real geometric transforms such as inevitable non-linear geometric distortion in the HSI imaging process, so this registration cannot accurately match two images. If we directly use this registration result to build the correspondences between HSI and HRCI, some corresponding pixel pairs may have very different appearances. However, after the coarse registration, it is with very large probability to find an accurate matching pixel near the current matching one. Therefore, in the fine matching stage, we use neighborhood searching to find the accurate matching pixels.

Given a set of user defined or randomly selected pixels in HSI, we attempt to find their corresponding pixels in the HRCI. Consider a pixel $(x, y)$ in the HSI, we first calculate its coarse corresponding point $\left(x^{\prime}, y^{\prime}\right)$ by the transform function $\mathbf{H}$. Then we performs a finer search to find the exact corresponding pixel $\left(u^{\prime}, v^{\prime}\right)$ in the neighborhood of $\left(x^{\prime}, y^{\prime}\right)$ according to the SIFT similarity. One benefit of manifold alignment is that, only a few corresponding pairs are required to transfer knowledge between the two manifolds, so that exact registration between 
HSI and HRCI is not necessary, which is very helpful for real applications.

After the correspondences are found, the joint adjacency matrix $W$ is constructed in step 3. Step 4 projects and aligns HSI and HRCI to a common embedding three-dimensional space. We denote the HSI and HRCI in the common space as $S_{c}=S F_{s}$ and $T_{c}=T F_{t}$ respectively. In step 5, the visualized HSI is acquired by mapping $S_{c}$ in the embedding space back to RGB space. Since $F_{t}$ is a linear mapping function from RGB space to the three-dimensional embedding space, its inverse function can map the image in the embedding space back to RGB space. The visualized HSI $S_{r g b}$ can be obtained by

$$
S_{r g b}=S_{c} F_{t}^{-1}=S F_{s} F_{t}^{-1}
$$

The proposed visualization method not only transfers color from HRCI to the visualized HSI, but also fuses the geometric structures of HSI and HRCI into the visualized result.

It is worth to mention that, the loss function in Equation (6) only preserves local geometries but not the global geometries of the manifold. In other words, it only encourages similar pixels in the original space to be neighbors in the embedding space, but does not prevent dissimilar pixels in the original space to be neighbors in the embedding space. However, by using linear manifold learning, the problem can be avoided in practice because two dissimilar pixels can always maintain their difference after linear transformation.

\section{EXPERIMENTS}

In this section, we evaluate the effectiveness of the proposed visualization algorithm on the remote sensing HSI and ground-based HSI, and compare it with three popularly used methods random band selection [3], PCA [6] and Laplacian Eigenmaps [7]. The impact of parameters (the number of corresponding pairs and the weight of the correspondence in the joint adjacency matrix $W$ ) on the performance is also discussed.

The first experiment is on a real remote sensing HSI taken over Washington D.C. mall by the hyperspectral digital imagery collection experiment (HYDICE) sensor. The data consists of 191 bands with the size of $1208 \times 307$. Fig. 2 displays the data by selecting three bands $(1,95,191)$ and mapping them to RGB space. The random band selection ignores the different importance of each band. Fig. 3 shows the RGB image created by PCA. It is a global transformation, so that some important local structures are lost. Fig. 4 displays the HSI using Laplacian Eigenmaps. It preserves local information of HSI better than PCA. For PCA method, because many of the pixel's coordinates in the new three-dimensional space are negative, we translate the coordinate axis to get positive values by subtracting each value with the minimum of the data. Then the positive data are globally and linearly mapped to the range of $0-255$ for display. For Laplacian Eigenmaps method, after projecting the HSI data to three-dimensional space, we map them to RGB space in the same way as what has been done to PCA. However, all of these methods use the false color to represent the materials in the scene, which brings poor visual effects and makes the image hard for human to understand. For example, the grass are displayed with red color and the buildings are displayed with green color, which contradicts with human normal understanding.

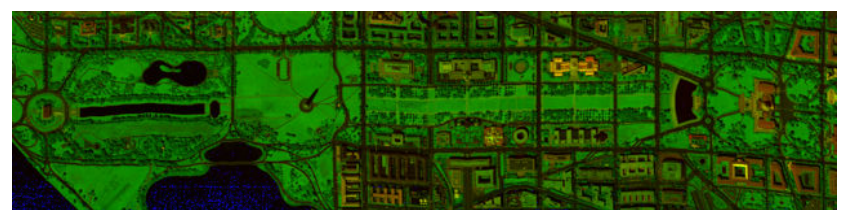

Fig. 2. HSI visualization via band selection, band 1, band 95 and band 191 .

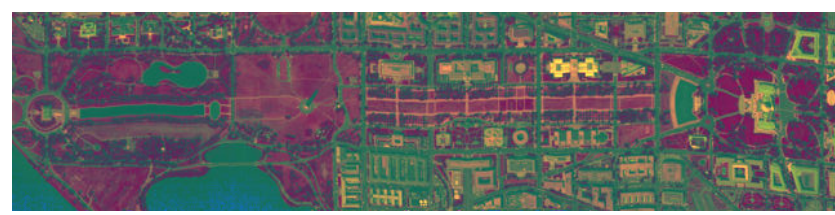

Fig. 3. HSI visualization via PCA

In our method, the HRCI of Washington DC Mall was obtained from Google maps with a high spatial resolution of $5160 \times 1640$, which is shown in Fig. 5. In this experiment, we randomly selected 20 pixels in HSI, and then found their corresponding pixels in the HRCI by SIFT feature matching. In manifold alignment algorithm, the parameters $\alpha_{1}$ and $\alpha_{2}$ were set to 1 and 500 respectively. Fig. 6 and Fig. 7 present the data of HSI and HRCI in the common embedding space as images. These two images have high similarity in color, indicating that two manifolds are successfully aligned. After the HSI in the embedding space are mapped back to RGB space, the visualized HSI image in Fig. 8 can be obtained. This image is similar to the HRCI in color, which make it easier for human to comprehend. Also, other two main goals of HSI visualization: consistent rendering and edge preservation are successfully reached. Consistent rendering claims that a spectra is always displayed as the same color value so that it can be easily recognized across different positions, and edge preservation demands that the edges of the original HSI are represented faithfully in the visualization. This success mainly relies on manifold alignment that not only fuses the HRCI information into visualization, but also retains the inherit structure of HSI.

Now we turn to study how the parameters affect the performance of the proposed approach and how to set suitable parameters to achieve a reasonable visualization result. In the proposed algorithm, if we fix $\alpha_{1}=1$, there are only two

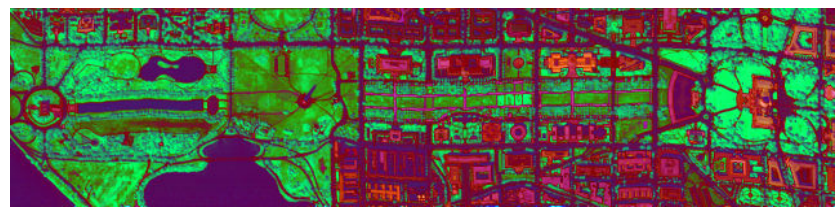

Fig. 4. HSI visualization via Laplacian Eigenmaps.

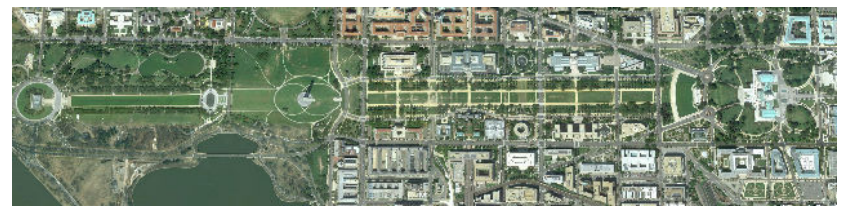

Fig. 5. HRCI on the same spot as HSI remote sensing data. 


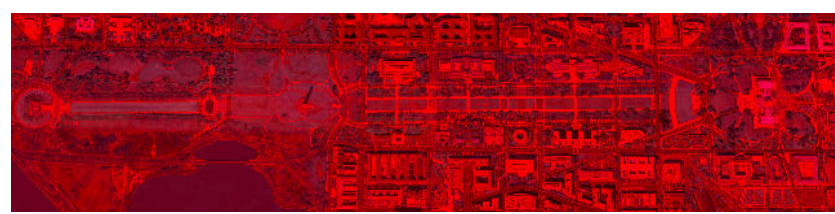

Fig. 6. HRCI projected to the common embedding space.

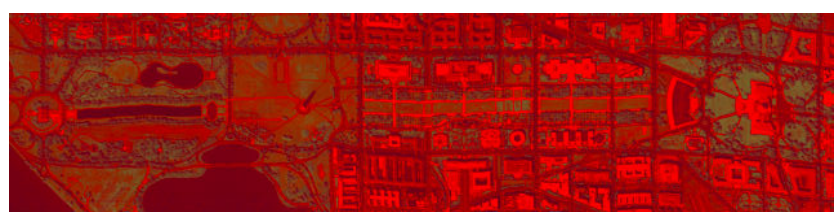

Fig. 7. HSI projected to the common embedding space.

free parameters, which are the number of corresponding pairs, and the weighting factor $\alpha_{2}$ to control the alignment. To analyze the impact of $\alpha_{2}$, we set up $\alpha_{2}=30,100,500$, and the number of the corresponding pairs is set to 20. Fig. 9 displays the visualized HSI corresponding to different values of $\alpha_{2}$. From this figure we observed that, as $\alpha_{2}$ becomes larger, the visualized HSI become more similar in color with the HRCI. However, when $\alpha_{2}$ is very large, the role of the corresponding pairs becomes to be very critical. In this case, if a few corresponding pairs are not correctly matched, the color transfer will produce very large error. Therefore, based on the experiments, we can always achieve reasonable results by setting $\alpha_{2}$ to be $200-500$.

Next we analyze the impact of the number of corresponding pairs on the performance of visualization. In this experiment, we fix $\alpha_{1}=1$ and $\alpha_{2}=500$. Fig. 10(a) is the result by randomly selecting 5 pixels in the HSI and then using SIFT feature to find their corresponding pairs in the HRCI. We observed that only 5 corresponding pairs can make the visualized image appears very naturally. When the number of corresponding pair is set to 30 , the visualization result is shown in Fig. 10(b), in which the color is very similar to the HRCI. In fact, the two parameters we discussed above have some relations. In the experiments, we have found that when the corresponding pair number become larger, $\alpha_{2}$ should be turned down to achieve reasonable results. This is probably because a balance between preserving each manifold's structure and aligning two manifolds (which is controlled by $\alpha_{2}$ and the number of corresponding pairs) is needed when creating the joint adjacency matrix $W$ of manifold alignment.

In order to further evaluate the proposed method, other two HSI data acquired by ground-based HSI camera were used in the experiments. Their corresponding HRCI data were acquired by traditional SLR digital camera. The HSI data have

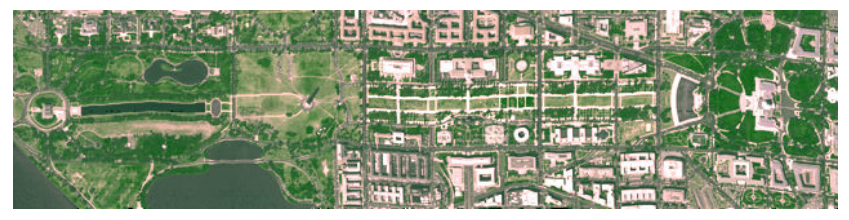

Fig. 8. Visualization result by the proposed method.

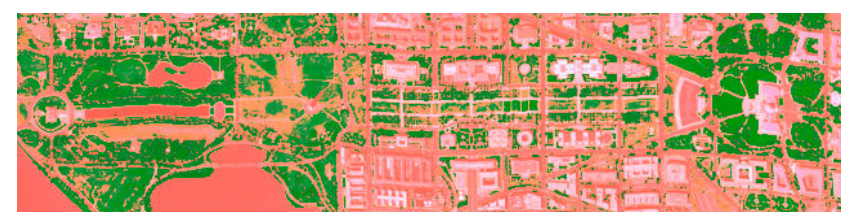

(a) $\alpha_{1}=1, \alpha_{2}=30$

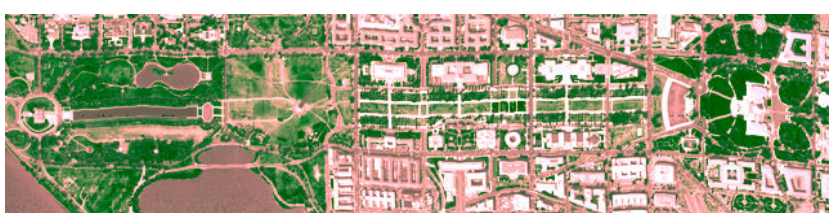

(b) $\alpha_{1}=1, \alpha_{2}=100$

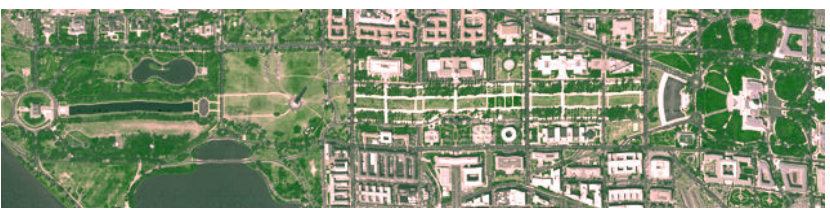

(c) $\alpha_{1}=1, \alpha_{2}=500$

Fig. 9. The impact of correspondence weight on the proposed visualization algorithm.

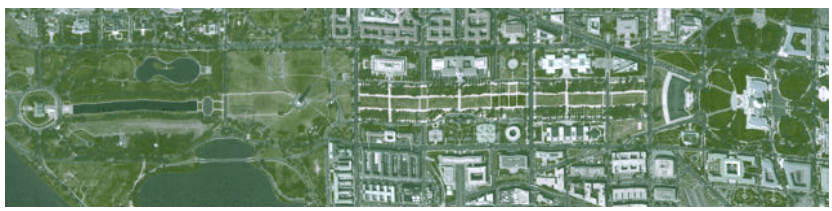

(a) corresponding pair number $=5$

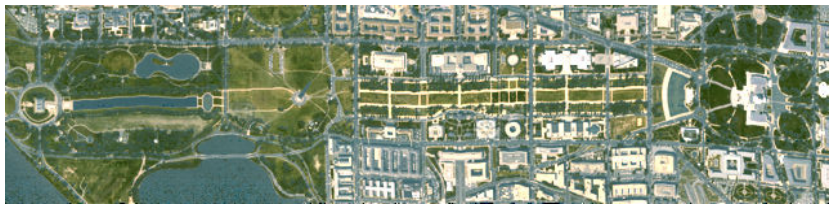

(b) corresponding pair number $=30$

Fig. 10. The impact of corresponding pair number on the proposed visualization algorithm.

a resolution of $480 \times 640 \times 18$, and the HRCI data have a resolution of $2592 \times 3872 \times 3$. The results of random band selection (here 1th,10th,18th bands are selected), PCA, eigenmaps, and manifold alignment methods $\left(\alpha_{1}=1, \alpha_{2}=300\right.$, corresponding pair number=20) are given in Fig. 11. The experimental results demonstrate that the proposed method is a very effective HSI visualization technique.

\section{CONClusions}

This paper presents a new approach to visualize HSI in natural color. Based on manifold alignment between HSI and HRCI, the proposed algorithm is able to find a projection function that maps the HSI directly from high-dimensional space to RGB space. The corresponding pixels between the HSI and the HRCI act as a bond to transfer the color information as well as local geometric information from the HRCI to HSI. The main advantage of the proposed algorithm is that only a few corresponding pairs are required to achieve a satisfied result. In other words, no exact image registration 


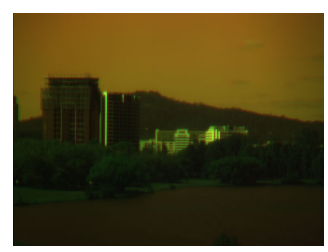

(a) Band selection

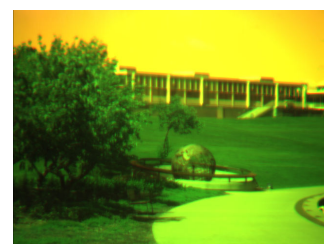

(f) Band selection

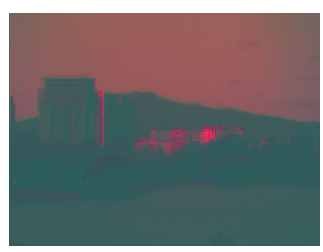

(b) PCA

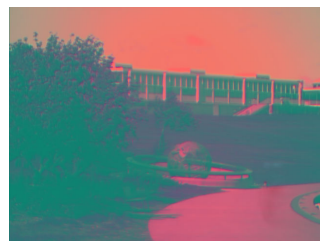

(g) PCA

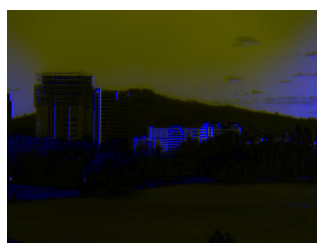

(c) Laplacian Eigenmaps

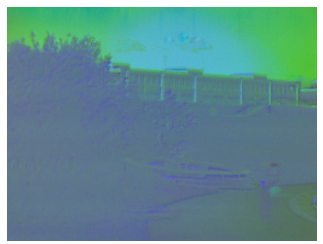

(h) Laplacian Eigenmaps

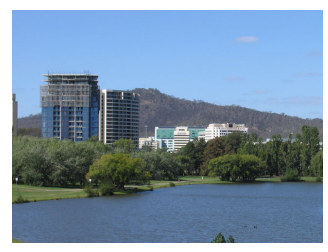

(d) Corresponding HRCI

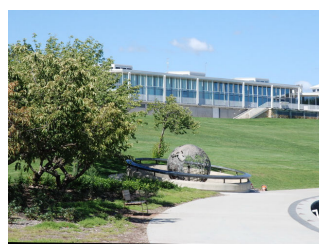

(i) Corresponding HRCI

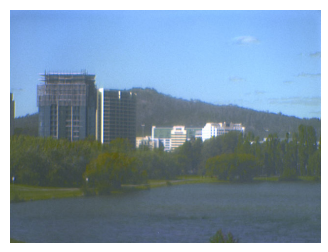

(e) Manifold alignment

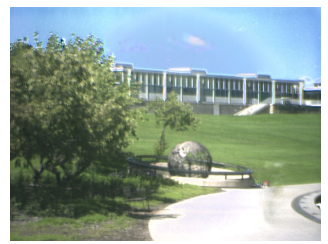

(j) Manifold alignment

Fig. 11. The visualization results on two HSI data captured by ground-based

is needed to find the corresponding pairs. By projecting the HSI directly to RGB space, our algorithm also avoid spectral distances distortion that other methods suffered while adjusting data range to the dynamic range of the display. One future work is to visualize HSI by align it with the HRCI from other spots which share similar features with the spot where the HSI was captured. Another work will focus on developing an interactive framework that allow users to assign corresponding pairs between HSI and HRCI.

\section{ACKNOWLEDGMENT}

The National Basic Research Program of China under Grant 2012CB316400, the National Natural Science Foundation of China under Grant 61171151, and the Australian Research Councils DECRA Projects funding scheme under Grant ID DE120102948 for funding this research.

\section{REFERENCES}

[1] Q. Du, N. Raksuntorn, S. Cai, and N. H. Younan, "Color representation and classification for hyperspectral imagery," in IEEE Int. Geosci. Remote Sens. Symp., 2006, pp. 537-540.

[2] N. Jacobson and M. Gupta, "Design goals and solutions for display of hyperspectral images," IEEE Trans. Geosci. Remote Sens., vol. 43, no. 11, pp. 2684-2692, 2005.

[3] MultiSpec. [Online], Available:http://cobweb.ecn.purdue.edu/biehl/ multispec/description.htm.

[4] Y. Qian, F. Yao, and S. Jia, "Band selection for hyperspectral imagery using affinity propagation," IET Comput. Vis., vol. 3, no. 4, pp. 213-222, 2009.

[5] HyperCube. [Online], Available:http://www.tec.army.mil/hypercube/.

[6] J. S. Tyo, A. Konsolakis, D. I. Diersen, and R. C. Olsen, "Principalcomponents-based display strategy for spectral imagery," IEEE Trans. Geosci. Remote Sens., vol. 41, no. 3, pp. 708-718, 2003.

[7] M. Belkin and P. Niyogi, "Laplacian eigenmaps for dimensionality reduction and data representation," Neural computation, vol. 15, no. 6, pp. 1373-1396, 2003.

[8] M. Mignotte, "A multiresolution markovian fusion model for the color visualization of hyperspectral images," IEEE Trans. Geosci. Remote Sens., vol. 48, no. 12, pp. 4236-4247, 2010.

[9] M. Cui, A. Razdan, J. Hu, and P. Wonka, "Interactive hyperspectral image visualization using convex optimization," IEEE Trans. Geosci. Remote Sens., vol. 47, no. 6, pp. 1673-1684, 2009.
[10] K. Kotwal and S. Chaudhuri, "Visualization of hyperspectral images using bilateral filtering," IEEE Trans. Geosci. Remote Sens., vol. 48, no. 5, pp. 2308-2316, 2010.

[11] C. Xu, D. Tao, and C. Xu, "A survey on multi-view learning," arXiv preprint arXiv:1304.5634, 2013.

[12] T.-M. Tu, P. S. Huang, C.-L. Hung, and C.-P. Chang, "A fast intensityhue-saturation fusion technique with spectral adjustment for ikonos imagery," IEEE Geosci. Remote Sens. Lett., vol. 1, no. 4, pp. 309-312, 2004.

[13] L. Ming and W. Shunjun, "A new image fusion algorithm based on wavelet transform," in Fifth Int. Conf. Computational Intelligence and Multimedia Applications. IEEE, 2003, pp. 154-159.

[14] Z. Wang, D. Ziou, C. Armenakis, D. Li, and Q. Li, "A comparative analysis of image fusion methods," IEEE Trans. Geosci. Remote Sens., vol. 43, no. 6, pp. 1391-1402, 2005.

[15] L. Alparone, L. Wald, J. Chanussot, C. Thomas, P. Gamba, and L. M. Bruce, "Comparison of pansharpening algorithms: Outcome of the 2006 grs-s data-fusion contest," IEEE Trans. Geosci. Remote Sens., vol. 45, no. 10, pp. 3012-3021, 2007.

[16] Y. Qian, D. Liao, and J. Zhou, "Manifold alignment based color transfer for multiview image stitching," in IEEE Int. Conf. on Image Processing, 2013.

[17] J. Ham, D. Lee, and L. Saul, "Semisupervised alignment of manifolds," in the Annual Conference on Uncertainty in Artificial Intelligence, vol. 10, 2005, pp. 120-127.

[18] C. Wang and S. Mahadevan, "A general framework for manifold alignment," in AAAI Fall Symposium on Manifold Learning and its Applications, 2009, pp. 53-58.

[19] D. Lowe, "Distinctive image features from scale-invariant keypoints," Int. J. Comput. Vis., vol. 60, no. 2, pp. 91-110, 2004.

[20] R. Hartley and A. Zisserman, Multiple view geometry in computer vision. Cambridge Univ. Press, 2000, vol. 2. 